\section{L'Actualité économique}

L'ACTUALITÉ ÉCONOMIQUE

Les options commerciales du Canada, par R.J. WONNACOTT. CONSEIL ÉCONOMIQUE DU CANADA, Ottawa, 1975.

\title{
Jacques Henry
}

Volume 53, numéro 1, janvier-mars 1977

URI : https://id.erudit.org/iderudit/800718ar

DOI : https://doi.org/10.7202/800718ar

Aller au sommaire du numéro

Éditeur(s)

HEC Montréal

ISSN

0001-771X (imprimé)

1710-3991 (numérique)

Découvrir la revue

Citer ce compte rendu

Henry, J. (1977). Compte rendu de [Les options commerciales du Canada, par R.J.

WONNACOTT. - CONSEIL ÉCONOMIQUE DU CANADA, Ottawa, 1975.]

L'Actualité économique, 53(1), 117-119. https://doi.org/10.7202/800718ar d'utilisation que vous pouvez consulter en ligne.

https://apropos.erudit.org/fr/usagers/politique-dutilisation/ 
Les options commerciales du Canada, par R.J. WonnaCotT. - CoNSEIL ECONOMIQUe dU CANADA, Ottawa, 1975.

L'étude de R.J. Wonnacott ne se présente pas comme un pur exercice académique visant à éclairer les options commerciales théoriquement accessibles à un pays quelconque à une époque indéterminée, mais elle est plutôt présentée comme une étude empirique qui aspire à déboucher sur la formulation de politiques concrètes pour le Canada des années 1970 et 1980 . Ne devant, au départ, être qu'un bref tour d'horizon d'études empiriques antérieures afin d'en tirer le plus grand nombre d'enseignements possibles (p. XIII), l'étude de Wonnacott s'est transformée en une analyse pseudoempirique de certaines options commerciales qui laisse complètement de côté les aspects non superficiels de la réalité commerciale, qui sont bien entendu ceux qui occasionnent les plus sérieuses difficultés d'appréciation lorsqu'il faut passer au stade qui consiste à définir en détail des politiques concrètes. Ces trois critiques appellent quelques explications.

Ce n'est pas tellement l'orientation générale de la politique commerciale canadienne qu'il est important de préciser : l'on ne trouvera aucun spécialiste de nos jours pour contester sérieusement l'objectif général d'une libéralisation plus poussée des échanges commerciaux à l'échelle mondiale, fondé sur la certitude qu'il en résultera des gains collectifs substantiels que les macro-agents pourront se partager. Disserter sur l'identification et la nature de l'objectif, comme Wonnacott le fait dans la majeure partie de son étude, c'est se limiter aux aspects superficiels du problème et ne rien ajouter de substantiel à ce qu'Adam Smith nous a démontré il y a exactement deux cents ans. Il saute aux yeux que le Canada «est un assez petit pays ayant pour voisin un géant économique » (p. 25), et personne ne contestera que «le Canada ne peut se permettre de se retrouver isolé dans un monde où les échanges seraient libérés 》(p. XVIII). L'on peut même admettre que la première citation justifie de poursuivre l'analyse en faisant l'hypothèse du «petit pays», ce qui autorise, d'une part, à conclure que, puisque le tarif américain contribue dans sa totalité à la détérioration des termes canadiens de l'échange (parce que c'est l'exportateur canadien qui le paie), son prélèvement par nos voisins du sud n'est vraisemblablement que pure méchanceté de leur part, et à souligner, d'autre part, que le prélèvement d'un tarif par le Canada relève du pur masochisme, car c'est le consommateur canadien qui le paie en entier, ce qui aggrave le mal au lieu de le guérir (p. 56-57), puisque l'effet conjugué des deux tarifs est de forcer les Canadiens à produire en se privant des économies d'échelle qu'ils pourraient réaliser s'ils avaient accès à un marché plus vaste, d'où inefficacité au niveau 
de la production et prix élevés aux consommateurs. La seconde citation, qui véhicule une idée plus vraie si l'on prend soin de remplacer le mot « libérés 》 par le qualificatif «bloqués», repose sur la notion très juste que l'échange international n'est en dernière analyse qu'un emprunt réciproque de marchés nationaux qui permet à chaque pays de développer plus efficacement sa capacité de production en empruntant pour ainsi dire les dimensions des autres pays ; or, comme les Canadiens ont eu la malencontreuse idée de s'établir là où leurs marchés «naturels » ne sont pas (p. 10), le Ganada est un pays avancé qui est dépendant des marchés étrangers (i.e. du marché américain) auxquels il se doit de gagner accès par une libéralisation des échanges.

Tout ce qui vient d'être dit ne contient aucune surprise. Mais c'est là où l'analyse de Wonnacott se termine que les difficultés se posent. Réglerat-on le problème de la dépendance canadienne (le mot est utilisé par Wonnacott à la page XXV) par une simple libéralisation des échanges ? Gagner un meilleur accès à l'étroit marché canadien qu'ils dominent déjà peut-il inciter les Américains à nous céder un meilleur accès à leur vaste marché que nous n'avons jusqu'ici réussi à pénétrer efficacement qu'avec des ententes très particulières de «libéralisation》, excepté bien entendu dans le secteur de la production primaire? Bien qu'il soit légitime de vouloir faire d'une pierre deux coups et même si l'on accepte dès le départ que c'est l'existence du tarif national qui explique la domination américaine de l'économie canadienne, peut-on raisonnablement supposer qu'une libéralisation pure et simple des échanges canado-américains (et non pas une libéralisation 《dirty 》), même assortie de mesures «temporaires 》 d'ajustement que Wonnacott se garde bien de définir, mettra fin à cette domination «paradoxale » du nord par le sud et amorcera la pénétration du marché sudiste par ces mêmes nordistes qui n'ont jamais pu dominer leur propre marché, même à l'abri d'une panoplie de mesures protectionnistes ? Répondre à cette question par un oui retentissant, c'est réduire la réalité commerciale à ses seules dimensions néoclassiques et remplacer l'argument protectionniste de l'industrie naissante par l'argument libéralisant de la pénétration naissante qu'il suffit d'amorcer, comme la pompe dans l'analogie macroéconomique.

Quant au caractère pseudo-empirique de l'étude, il se manifeste dans le fait que seules quelques estimations quantitatives datant déjà de quelques années y sont présentées, et elles éclairent beaucoup plus l'orientation générale de la politique commerciale que le degré d'intensité de la libéralisation à réaliser ou les distinctions sectorielles qu'il conviendrait de faire ; pour le reste, on ne trouve dans la majeure partie de l'étude que des guestimations qualitatives. Ainsi, le classement suggéré des options commerciales retenues (p. $\mathrm{XX}$ ), tout en cherchant à véhiculer l'impression d'un degré élevé de précision empirique, n'est au fond qu'un classement ordinal qui n'éclaire que très sommairement les avantages économiques éventuels pour le $\mathrm{Ca}$ nada. De telles comparaisons «approximatives» (p. 80) et estimations «à vue » (p. 167) sont sans doute légitimes lorsqu'elles sont présentées au tableau noir, mais elles sont complètement inadéquates dans une étude qui entend déboucher sur des prescriptions politiques précises. 
Enfin, toutes les options commerciales qui s'offrent actuellement au Canada n'y sont pas abordées avec le même sérieux : seules sont effectivement retenues les options qui, tout en ne s'éloignant pas trop de la situation actuelle, possèdent l'avantage d'être plus faciles à analyser, comme l'auteur l'avoue lui-même (p. 234).

Le professeur Wonnacott se savait sans doute vulnérable aux critiques bien évidentes que je viens de formuler : il admet lui-même que «ses conclusions sont entachées d'une forte marge d'erreur sans que nous sachions dans quel sens » (p. 100), ce qui définit en général la situation dans laquelle l'économiste se trouve avant même de s'attaquer empiriquement à un problème. L'auteur reconnaît qu'il eut fallu se livrer à une analyse plus complexe pour répondre adéquatement aux questions posées. Or, c'est précisément ce type d'analyse - dont Wonnacott est capable - qu'il eut fallu faire.

L'idéal du libre-échange multilatéral (LEM) sur lequel Wonnacott propose d'aligner la politique commercaile du Canada repose sur de solides fondements scientifiques dans un monde de marchés parfaits et d'agents indifférents. Cependant, dans un monde de marchés imparfaits caractérisé par la présence de multiples effets externes et d'inégalités souvent croissantes entre des agents dont on peut sérieusement tout dire, sauf qu'ils sont indifférents les uns aux autres, il faut savoir ne pas abuser de la notion de «liberté ». Le principe de la liberté des échanges commerciaux ne délégitime pas ipso facto toute politique commerciale dite «restrictive», pas plus que l'idée (émise par N. Georgescu-Roegen à la conférence Dai Dong à Stockholm en 1972) qu'il est plus facile de redistribuer la population mondiale que de redistribuer les ressources mondiales ne délégitime les politiques d'immigration (qui sont, par définition, « restrictives»), et pas davantage que le principe sacré de la liberté de choix de la langue ne délégitime la loi 22 (ou toute autre loi visant le même objectif). Erigée en système absolu, la liberté ne peut que répartir les avantages qu'elle ne confère que proportionnellement à la taille et au pouvoir des agents économiques.

Jacques HENRY, Université d'Ottawa 